\title{
Résultats canadiens de l'Enquête européenne en ligne sur la sexualité entre hommes (EMIS-2017)
}

\author{
N Brogan ${ }^{1}$, DM Paquette ${ }^{*}$, NJ Lachowsky², M Blais ${ }^{3}$, DJ Brennan4 ${ }^{4}$ TA Hart ${ }^{5}$, B Adam ${ }^{6,7}$
}

\section{Résumé}

Contexte : En 2017, l'Enquête européenne en ligne sur la sexualité entre hommes (EMIS-2017) a permis de recueillir des données dans 50 pays, y compris le Canada pour la première fois.

Objectif : Fournir un aperçu des données canadiennes de l'EMIS-2017, en vue de décrire les besoins associés aux infections transmissibles sexuellement et par le sang (ITSS) des hommes gais, bisexuels et autres hommes ayant des relations sexuelles avec des hommes (gbHARSAH).

Méthodes : Le questionnaire de l'EMIS-2017 était une version mise à jour de l'EMIS-2010. II incluait des données auto-déclarées sociodémographiques ainsi que relatives à toute expérience de discrimination, à la santé mentale et à l'usage de substance, à la connaissance de la prophylaxie préexposition (PrEP) pour le VIH, aux pratiques sexuelles ainsi qu'à l'historique de dépistage et de diagnostic d'ITSS. L'analyse a été principalement descriptive.

Résultats : Sur 6059 répondants au Canada, 5165 participants ont répondu aux critères d'inclusion à cette analyse. La majorité des participants étaient nés au Canada $(79,3 \%)$ et plus de la moitié d'entre eux $(56,7 \%)$ étaient âgés de moins de 39 ans. En termes de discrimination associée à leur attirance pour d'autres hommes, les participants ont déclaré un niveau élevé d'intimidation (31,9\%), de la violence verbale $(22,1 \%)$ et de la violence physique $(1,5 \%)$ au cours de l'année précédente. En matière de santé mentale, $23,9 \%$ enregistraient un résultat modéré à grave de dépression ou d'anxiété. Près des deux tiers (64,1 \%) signalaient un usage de substances et un cinquième $(21,5 \%)$ déclaraient le recours au chemsex (c.-à-d. I'utilisation de drogues stimulantes pour rendre l'expérience sexuelle plus intense ou la faire durer plus longtemps). Seuls 8,4 \% des participants ont signalé l'utilisation de la PrEP pour le $\mathrm{VIH}$; toutefois, 51,7 \% ont indiqué être enclins à utiliser la PrEP, si elle était disponible et abordable. Les pratiques sexuelles, comme l'utilisation d'un préservatif, variaient en fonction de la PrEP utilisée; 91,3\% des hommes utilisant la PrEP indiquaient des pénétrations anales sans préservatif (PASP), par rapport à 71,5\% des hommes n'utilisant pas la PrEP. En ce qui concerne le dépistage d'ITSS, 1,5\% a signalé avoir reçu un diagnostic d'hépatite $\mathrm{C}$ et $9,0 \%$, un diagnostic de $\mathrm{VIH}$. Parmi ceux ayant reçu un diagnostic de $\mathrm{VIH}$, la plupart suivaient un traitement $(99,1 \%)$ et avaient une charge virale indétectable $(96,7 \%)$.

Conclusion : Les hommes gbHARSAH au Canada ont fait l'objet d'une stigmatisation, de discrimination et souffrent de troubles mentaux; I'usage de substances était élevé, tout comme les pratiques sexuelles à risque élevé, comme des PASP, parmi certains groupes d'hommes. Un écart existait entre la proportion des hommes intéressés par la PrEP et ceux I'utilisant effectivement; le dépistage complet d'ITSS était faible.

Ces résultats peuvent aider les mesures de santé publique et fournir une base d'examen de l'incidence d'interventions actuelles et nouvelles.

Citation proposée : Brogan N, Paquette DM, Lachowsky NJ, Blais M, Brennan DJ, Hart TA, Adam B. Résultats canadiens de l'Enquête européenne en ligne sur la sexualité entre hommes (EMIS-2017). Relevé des maladies transmissibles au Canada 2019;45(11):298-310. https://doi.org/10.14745/ccdr.v45i11a01f

Mots-clés : $\mathrm{VIH}$, infections transmissibles sexuellement, pratiques sexuelles à risque, hommes gais, bisexuels et autres hommes ayant des relations sexuelles avec des hommes, gbHARSAH, prophylaxie préexposition, prophylaxie postexposition
Cette oeuvre est mise à la disposition selon les termes de la licence internationale Creative Commons Attribution 4.0

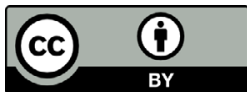

Affiliations

${ }^{1}$ Agence de la santé publique du Canada, Ottawa, ON

2 Université de Victoria, Victoria, BC

${ }^{3}$ Université du Québec à Montréal, Montréal, QC

${ }^{4}$ Université de Toronto, Toronto, ON

${ }^{5}$ Université Ryerson et Université de Toronto, Toronto, ON

${ }^{6}$ Université de Windsor, Windsor, ON

${ }^{7}$ Ontario HIV Treatment Network, Toronto, ON

\section{*Correspondance:}

dana.paquette@canada.ca 


\section{Introduction}

En 2017, la deuxième itération de l'Enquête européenne en ligne sur la sexualité entre hommes (EMIS-2017) a été lancée (1). Dans le cadre de cette enquête, des renseignements ont été recueillis auprès d'hommes gais, bisexuels et d'autres hommes ayant des relations sexuelles avec des hommes (gbHARSAH) vivant dans 50 pays, dans le but de générer des données utiles à la planification de la prévention du VIH et d'infections transmissibles sexuellement (ITS) ainsi que de programmes de soins en la matière et au suivi des progrès dans ces domaines (1). Le Canada a participé à cette enquête pour la première fois.

Au Canada, les hommes gbHARSAH continuent d'être particulièrement touchés par les infections transmissibles sexuellement et par le sang (ITSS). En 2016, il a été estimé que les hommes gbHARSAH représentaient plus de la moitié $(52,5 \%)$ de la population vivant avec une infection à VIH au Canada (2). Les taux de syphilis et de gonorrhée ont augmenté au cours des dernières années; cette hausse chez les hommes ayant surtout lieu au sein de la population des hommes gbHARSAH au Canada (3-5) et internationalement $(6,7)$. L'hépatite $C$ est également en hausse chez les hommes gbHARSAH séropositifs au VIH (8).

Les raisons pour cela sont multifactorielles. Parmi les hommes séronégatifs au $\mathrm{VIH}$, de nouvelles interventions préventives, comme la prophylaxie préexposition (PrEP), associées à une réduction de l'utilisation des préservatifs peuvent, en partie, contribuer à l'augmentation des ITS autres que le VIH $(3,4)$. Parmi les hommes séropositifs au $\mathrm{VIH}$, le sérotriage et la connaissance d'une charge virale indétectable peuvent réduire le risque de transmission du $\mathrm{VIH}$, qui peut entraîner une diminution simultanée de l'utilisation de préservatifs, augmentant ainsi le risque d'autres ITSS $(3,4)$. D'autres facteurs pouvant influencer les taux d'ITSS comprennent des normes communautaires changeantes et l'utilisation de drogues illicites pour intensifier les expériences sexuelles (c.-à-d. le chemsex) (9). Des facteurs structurels, comme la stigmatisation associée à l'orientation sexuelle et l'infection à $\mathrm{VIH}$, un manque de connaissance et de formation des prestataires quant aux risques et aux besoins en matière de santé sexuelle des hommes gbHARSAH ainsi que des problèmes liés aux coûts et à l'accès à la PrEP et au traitement contre le VIH, peuvent également contribuer à la charge des ITSS chez les hommes gbHARSAH (10).

Rassembler des renseignements au niveau national sur les comportements à risque et les comportements de prévention, les besoins en services de santé ainsi que les résultats en matière de santé est essentiel pour comprendre les tendances actuelles et orienter la planification et l'évaluation des interventions de santé publique visant à prévenir les ITSS chez les hommes
gbHARSAH. L'objectif de ce rapport est de présenter un aperçu des données de l'EMIS-2017 pour le Canada

\section{Méthodes}

L'EMIS-2017 a été menée par Sigma Research à la London School of Hygiene and Tropical Medicine en association avec I'Institut Robert-Koch de Berlin. L'enquête a été financée par le Programme-Santé de I' Union européenne 2014-2020 pour l'Europe. L'enquête s'est déroulée d'octobre 2017 à janvier 2018, inclusivement, dans 50 pays. Au Canada, l'Agence de la santé publique du Canada (ASPC) a financé la promotion de l'enquête auprès des hommes gbHARSAH canadiens. Des chercheurs communautaires (N. J. L., M. B., D. J. B., T. A. H. et B. A.) ainsi que deux représentants de l'ASPC ont fourni des commentaires sur le questionnaire et les méthodes de recrutement et ont examiné et interprété les résultats.

Alors que l'EMIS utilise le terme " homme ayant des relations sexuelles avec des hommes (HARSAH) », les auteurs utilisent quant à eux celui d'hommes " gbHARSAH » pour décrire la même population puisque ce dernier est couramment utilisé au Canada. Une description plus détaillée des méthodes est disponible ailleurs (11).

\section{Questionnaire}

L'enquête EMIS-2017 est fondée sur le questionnaire utilisé avec succès lors de l'EMIS-2010. Les mises à jour ont reflété un examen des données épidémiologiques de l'infection à $\mathrm{VIH}$ et des ITS, les comportements à risque et préventifs associés aux ITSS chez les hommes gbHARSAH, un exercice d'inventaire des politiques et des pratiques, un exercice d'évaluation de la portée des questionnaires disponibles sur les hommes gbHARSAH publiés depuis l'EMIS-2010 et trois séries de consultations auprès des pays partenaires, notamment le Canada.

La version finale du questionnaire a inclus des questions sur :

- les caractéristiques sociodémographiques des participants à l'EMIS

- les expériences en matière de discrimination, de santé mentale et d'usage de substances

- la connaissance et l'utilisation de la prophylaxie postexposition (PEP) et préexposition (PrEP)

- les pratiques sexuelles (les renseignements sur le chemsex ont été capturés en posant des questions sur l'utilisation de drogues stimulantes, notamment l'ecstasy/MDMA, la cocaïne, l'amphétamine, la crystal méthamphétamine, la méphédrone et la kétamine afin de rendre l'expérience sexuelle plus intense et la faire durer plus longtemps)

- le dépistage et de diagnostic d'ITSS 
L'enquête a été disponible simultanément dans 33 langues, notamment 22 des 23 langues officielles de l'Union européenne; les chercheurs canadiens ont apporté des modifications aux versions en français et en anglais et le questionnaire a été proposé en version pilote auprès d'un petit groupe d'hommes gbHARSAH canadiens. Le questionnaire de l'EMIS-2017 est accessible en ligne (12).

\section{Recrutement}

Le personnel de Sigma a délégué la publicité à dix plateformes de rencontre fonctionnant dans plusieurs pays, notamment PlanetRomeo, Grindr, Hornet, Oruiser, RECON, Scruff, Gaydar, Manhunt/Jack'd, GROWLr et Bluesystem. Au Canada, des publicités et bannières ont été publiées dans les réseaux sociaux, sur des sites Web d'actualités homosexuelles et dans des applications de réseautage sexuel. Le matériel promotionnel électronique et hors ligne a été fourni à des organismes communautaires dans l'ensemble du Canada, comme l'initiative Health Initiative for Men, aux fins de distribution au sein de leurs réseaux.

Une adresse URL particulière dirigeant les participants vers la page cible de l'EMIS a été donné, pour toutes les promotions en ligne de I'EMIS, payantes ou non. Ils pouvaient alors y faire leur sélection de langue et prendre part à l'enquête; cette page recueillait également des données sur la source de recrutement. Malheureusement, les codes sources de Grindr présentaient des étiquettes erronées au Canada; lors du nettoyage des données, elles ont été rectifiées le plus possible à l'aide du calendrier des invitations de participation envoyées par Grindr.

\section{Critères de consentement et d'inclusion}

Avant de prendre part à l'enquête, les participants au Canada devaient confirmer avoir lu et compris la nature et l'objet de l'étude, souhaiter participer et être âgés d'au moins 16 ans. Les critères d'inclusion comprenaient les suivants :

- vivre dans l'un des 50 pays participant à l'étude

- se considérer comme étant un homme, y compris les hommes cisgenres (c.-à-d. les personnes désignées comme étant des hommes à la naissance et se considérant comme étant des hommes) et les hommes transgenres (c.-à-d., les personnes désignés comme étant des femmes à la naissance et se considérant comme étant des hommes)

- être sexuellement attirés par des hommes et/ou avoir déjà eu des relations sexuelles avec des hommes

Aux fins de cette analyse, les participants devaient fournir la première partie de leur code postal, afin qu'une province ou un territoire puisse leur être attribué(e). Trois indicateurs de divergence ont été créés relativement à l'âge, aux partenaires masculins stables et aux partenaires occasionnels. Les participants fournissant des données incohérentes ont été exclus de l'analyse.

\section{Analyse}

Des analyses descriptives (fréquences et proportions) ont été menées. Les caractéristiques des participants ont été décrites par province et territoire. Du fait de tailles d'échantillon inférieures à 10, les hommes résidant au Yukon ont été combinés à ceux vivant en Colombie-Britannique; ceux des Territoires du Nord-Ouest à ceux vivant en Alberta et ceux vivant au Nunavut à ceux de l'Ontario. De la même façon, les participants du Manitoba et de la Saskatchewan ont été combinés, tout comme ceux des quatre provinces atlantiques.

Une mesure combinée d'anxiété et de dépression a été calculée à l'aide d'une échelle validée d'analyse rapide pour l'anxiété et la dépression : le Questionnaire sur la santé du patient-4 (13). Le questionnaire CAGE à 4 questions a été utilisé comme indicateur de dépendance à l'alcool (14).

Pour surveiller l'adoption et l'effet des nouvelles stratégies de prévention du VIH, les indicateurs suivants ont été dérivés :

- pénétration anale avec des partenaires occasionnels

- utilisation de préservatifs avec des partenaires occasionnels

- pénétration anale sans préservatif avec des partenaires occasionnels au cours des douze mois précédents par des hommes séropositifs au $\mathrm{VIH}$ suivant un traitement contre le $\mathrm{VIH}$ et ayant une charge virale indétectable

- pénétration anale sans préservatif avec des partenaires occasionnels au cours des douze mois précédents par des hommes séronégatifs utilisant la PrEP

- pénétration anale sans préservatif avec des partenaires occasionnels au cours des douze mois précédents par des hommes séropositifs au VIH ne suivant pas de traitement contre le VIH ou ayant une charge virale détectable

- pénétration anale sans préservatif avec des partenaires occasionnels au cours des douze mois précédents par des hommes séronégatifs ou n'ayant pas fait l'objet d'un dépistage et n'utilisant pas de PrEP (15). Dans le cadre du questionnaire, les partenaires occasionnels désignaient tout partenaire non défini comme un partenaire stable: " hommes avec lesquels vous avez eu des rapports sexuels une seule fois et des hommes avec lesquels vous avez eu des rapports sexuels plus d'une fois, mais que vous ne considérez pas comme partenaire stable »

Le " dépistage complet d'ITS » était une variable composite indiquant le dépistage d'une infection à $\mathrm{VIH}$, une analyse de sang ainsi qu'un prélèvement anal et urétral (ou encore un prélèvement vaginal et une analyse $d$ 'urine) chez les répondants n'ayant pas reçu de diagnostic de $\mathrm{VIH}$ au cours des 12 mois précédents. Seuls les répondants n'ayant pas reçu de diagnostic de VIH ont été inclus dans cet indicateur, afin de veiller à ce qu'il ne soit pas influencé par le nombre d'hommes ayant reçu un diagnostic de $\mathrm{VIH}$, qui font généralement des analyses routinières d'ITS dans le cadre de leur suivi clinique. Cette variable a été créée en vue d'une comparaison dans l'ensemble du pays quant au dépistage d'ITS et à la couverture des services 
de traitement conformément à la surveillance de l'application de la " déclaration de Dublin » (16). Même si des prélèvements pharyngés sont recommandés au Canada (17), I'EMIS-2017 n'a pas recueilli ce renseignement.

Les nombres et proportions ont été supprimés lorsque le numérateur était inférieur à cinq et lorsque le dénominateur était inférieur à cent.

\section{Résultats}

Au total, 6059 hommes gbHARSAH canadiens ont participé à I'EMIS-2017. Parmi eux, 894 (14,8\%) ont été retirés de l'enquête, du fait de données présentant des divergences ou ne comprenant pas la région de tri d'acheminement (c.-à-d. les trois premiers caractères du code postal) et empêchant un classement par province/territoire. Les 5165 participants restant pour le Canada ont été inclus dans cette analyse.

\section{Caractéristiques sociodémographiques des participants à I'EMIS}

Toutes les provinces et tous les territoires ont été représentés : 23,1\% pour la Colombie-Britannique et le Yukon, 13,7\% pour l'Alberta et les Territoires du Nord-Ouest, 6,6 \% pour la Saskatchewan et le Manitoba, 33,0 \% pour l'Ontario et le Nunavut, 15,3\% pour le Québec et 8,3\% pour les provinces atlantiques. Au niveau national, la majorité des participants ont répondu à l'enquête en anglais $(91,0 \%), 7,4 \%$ y ont répondu en français et le 1,6\% restant y a répondu dans l'une des 19 autres langues.

Plus de $50 \%$ des participants étaient âgés de moins de 39 ans (56,7 \%) (tableau 1). L'âge médian de la population était

Tableau 1 : Caractéristiques sociodémographiques des participants canadiens à l'EMIS-2017 $(\mathrm{N}=5,165)$

\begin{tabular}{|c|c|c|c|c|c|c|c|c|c|c|c|c|c|c|}
\hline \multirow[t]{2}{*}{ Caractéristiques } & \multicolumn{2}{|c|}{$\begin{array}{c}\text { Colombie- } \\
\text { Britannique + } \\
\text { Yukon } \\
(\mathrm{n}=1191)^{\mathrm{a}}\end{array}$} & \multicolumn{2}{|c|}{$\begin{array}{l}\text { Alberta + } \\
\text { Territoires du } \\
\text { Nord-Ouest } \\
(n=710)^{a}\end{array}$} & \multicolumn{2}{|c|}{$\begin{array}{c}\text { Saskatchewan } \\
+ \\
\text { Manitoba } \\
(n=339)^{\mathrm{a}}\end{array}$} & \multicolumn{2}{|c|}{$\begin{array}{c}\text { Ontario } \\
+ \\
\text { Nunavut } \\
(\mathrm{n}=1,707)^{\mathrm{a}}\end{array}$} & \multicolumn{2}{|c|}{$\begin{array}{l}\text { Québec } \\
(\mathrm{n}=789)^{\mathrm{a}}\end{array}$} & \multicolumn{2}{|c|}{$\begin{array}{c}\text { Provinces } \\
\text { atlantiques } \\
(n=429)^{a}\end{array}$} & \multicolumn{2}{|c|}{$\begin{array}{c}\text { Total } \\
(\mathrm{N}=5165)^{\mathrm{a}}\end{array}$} \\
\hline & $n$ & $\%$ & $n$ & $\%$ & $n$ & $\%$ & $n$ & $\%$ & $n$ & $\%$ & $\mathrm{n}$ & $\%$ & $\mathrm{n}$ & $\%$ \\
\hline \multicolumn{15}{|l|}{ Catégorie d'âge } \\
\hline 16 à 24 ans & 160 & 13,4 & 148 & 20,8 & 63 & 18,6 & 281 & 16,5 & 165 & 20,9 & 89 & 20,7 & 906 & 17,5 \\
\hline 25 à 39 ans & 492 & 41,3 & 295 & 41,5 & 137 & 40,4 & 607 & 35,6 & 334 & 42,3 & 161 & 37,5 & 2026 & 39,2 \\
\hline 40 à 54 ans & 326 & 27,4 & 166 & 23,4 & 83 & 24,5 & 526 & 30,8 & 179 & 22,7 & 96 & 22,4 & 1376 & 26,6 \\
\hline 55 à 69 ans & 193 & 16,2 & 90 & 12,7 & 53 & 15,6 & 250 & 14,6 & 102 & 12,9 & 77 & 17,9 & 765 & 14,8 \\
\hline Plus de 70 ans & 20 & 1,7 & 11 & 1,5 & 3 & 0,88 & 43 & 2,5 & 9 & 1,1 & 6 & 1,4 & 92 & 1,8 \\
\hline \multicolumn{15}{|l|}{ Identité sexuelle } \\
\hline Gay ou homosexuel & 975 & 82,0 & 503 & 70,8 & 223 & 65,8 & 1294 & 75,8 & 627 & 79,5 & 302 & 70,4 & 3924 & 76,0 \\
\hline Bisexuel & 138 & 11,6 & 156 & 22,0 & 98 & 28,9 & 297 & 17,4 & 116 & 14,7 & 96 & 22,4 & 901 & 17,5 \\
\hline Hétérosexuel & 4 & 0,3 & 12 & 1,7 & 6 & 1,8 & 17 & 1,0 & 4 & 0,5 & 2 & 0,5 & 45 & 0,9 \\
\hline Autre & 72 & 6,1 & 39 & 5,5 & 12 & 3,5 & 99 & 5,8 & 42 & 5,3 & 29 & 6,8 & 293 & 5,7 \\
\hline \multicolumn{15}{|l|}{ Identité de genre } \\
\hline Homme cisgenre & 1158 & 97,2 & 690 & 97,2 & 331 & 97,6 & 1675 & 98,1 & 772 & 97,8 & 413 & 96,3 & 5039 & 97,6 \\
\hline Homme transgenre & 33 & 2,8 & 20 & 2,8 & 8 & 2,4 & 32 & 1,9 & 17 & 2,2 & 16 & 3,7 & 126 & 2,4 \\
\hline \multicolumn{15}{|c|}{ Proportion d'amis et membres de la famille au courant de cette attirance par des hommes } \\
\hline Tous ou presque tous & 652 & 55,1 & 291 & 41,7 & 139 & 41,5 & 819 & 48,6 & 432 & 55,0 & 203 & 47,4 & 2536 & 49,6 \\
\hline Plus de la moitié & 227 & 19,2 & 125 & 17,9 & 52 & 15,5 & 318 & 18,9 & 124 & 15,8 & 60 & 14,0 & 906 & 17,7 \\
\hline Moins de la moitié & 136 & 11,5 & 80 & 11,5 & 44 & 13,1 & 182 & 10,8 & 74 & 9,4 & 54 & 12,6 & 570 & 11,1 \\
\hline Peu & 131 & 11,1 & 135 & 19,3 & 71 & 21,2 & 255 & 15,1 & 100 & 12,7 & 79 & 18,5 & 771 & 15,1 \\
\hline Aucun & 38 & 3,2 & 67 & 9,6 & 29 & 8,7 & 110 & 6,5 & 55 & 7,0 & 32 & 7,5 & 331 & 6,5 \\
\hline \multicolumn{15}{|l|}{ Né au Canada } \\
\hline Non & 307 & 25,8 & 99 & 14,0 & 38 & 11,2 & 396 & 23,2 & 190 & 24,1 & 40 & 9,3 & 1070 & 20,7 \\
\hline Oui & 881 & 74,2 & 610 & 86,0 & 301 & 88,8 & 1309 & 76,8 & 598 & 75,9 & 388 & 90,7 & 4087 & 79,3 \\
\hline \multicolumn{15}{|l|}{ Ethnicité } \\
\hline Blanc & 795 & 74,2 & 507 & 80,9 & 237 & 78,0 & 1151 & 76,5 & 524 & 78,9 & 306 & 87,7 & 3520 & 77,9 \\
\hline Autochtone & 46 & 4,3 & 34 & 5,4 & 24 & 7,9 & 35 & 2,3 & 20 & 3,0 & 11 & 3,2 & 170 & 3,8 \\
\hline Latino-américain & 39 & 3,6 & 16 & 2,6 & 10 & 3,3 & 53 & 3,5 & 41 & 6,2 & 1 & 0,3 & 160 & 3,5 \\
\hline Asiatique & 47 & 4,4 & 17 & 2,7 & 8 & 2,6 & 53 & 3,5 & 12 & 1,8 & 3 & 0,9 & 140 & 3,1 \\
\hline Est/sud-est-asiatique & 57 & 5,3 & 16 & 2,6 & 5 & 1,6 & 36 & 2,4 & 8 & 1,2 & 4 & 1,1 & 126 & 2,8 \\
\hline Sud-asiatique & 22 & 2,1 & 10 & 1,6 & 4 & 1,3 & 45 & 3,0 & 5 & 0,8 & 2 & 0,6 & 88 & 1,9 \\
\hline Noirb & 10 & 0,9 & 6 & 1,0 & 5 & 1,6 & 43 & 2,9 & 7 & 1,1 & 6 & 1,7 & 77 & 1,7 \\
\hline Arabe/asiatique de l'Ouest & 13 & 1,2 & 5 & 0,8 & 2 & 0,7 & 22 & 1,5 & 19 & 2,9 & 4 & 1,1 & 65 & 1,4 \\
\hline Autre & 42 & 3,9 & 16 & 2,6 & 9 & 3,0 & 67 & 4,5 & 28 & 4,2 & 12 & 3,4 & 174 & 3,8 \\
\hline
\end{tabular}


Tableau 1 : Caractéristiques sociodémographiques des participants canadiens à l'EMIS-2017 ( $N=5,165)$ (suite)

\begin{tabular}{|c|c|c|c|c|c|c|c|c|c|c|c|c|c|c|}
\hline \multirow[t]{2}{*}{ Caractéristiques } & \multicolumn{2}{|c|}{$\begin{array}{c}\text { Colombie- } \\
\text { Britannique + } \\
\text { Yukon } \\
(n=1191)^{\mathrm{a}}\end{array}$} & \multicolumn{2}{|c|}{$\begin{array}{l}\text { Alberta }+ \\
\text { Territoires du } \\
\text { Nord-Ouest } \\
(n=710)^{\mathrm{a}}\end{array}$} & \multicolumn{2}{|c|}{$\begin{array}{c}\text { Saskatchewan } \\
+ \\
\text { Manitoba } \\
(n=339)^{a}\end{array}$} & \multicolumn{2}{|c|}{$\begin{array}{c}\text { Ontario } \\
+ \\
\text { Nunavut } \\
(n=1707)^{\mathrm{a}}\end{array}$} & \multicolumn{2}{|c|}{$\begin{array}{l}\text { Québec } \\
(n=789)^{a}\end{array}$} & \multicolumn{2}{|c|}{$\begin{array}{c}\begin{array}{c}\text { Provinces } \\
\text { atlantiques }\end{array} \\
(n=429)^{a}\end{array}$} & \multicolumn{2}{|c|}{$\begin{array}{c}\text { Total } \\
(\mathrm{N}=5165)^{\mathrm{a}}\end{array}$} \\
\hline & $\mathrm{n}$ & $\%$ & $\mathrm{n}$ & $\%$ & $\mathrm{n}$ & $\%$ & n & $\%$ & $\mathbf{n}$ & $\%$ & $\mathbf{n}$ & $\%$ & $\mathbf{n}$ & $\%$ \\
\hline \multicolumn{15}{|c|}{ Années d'études après l'âge de 16 ans } \\
\hline Aucune & 113 & 9,5 & 70 & 9,9 & 37 & 10,9 & 174 & 10,2 & 50 & 6,3 & 39 & 9,1 & 483 & 9,4 \\
\hline 1 à 3 & 156 & 13,1 & 124 & 17,5 & 49 & 14,5 & 168 & 9,8 & 66 & 8,4 & 65 & 15,2 & 628 & 12,2 \\
\hline 4 à 6 & 322 & 27,0 & 210 & 29,6 & 116 & 34,2 & 479 & 28,1 & 193 & 24,5 & 136 & 31,7 & 1456 & 28,2 \\
\hline Plus de 7 ans & 600 & 50,4 & 306 & 43,1 & 137 & 40,4 & 886 & 51,9 & 480 & 60,8 & 189 & 44,1 & 2598 & 50,3 \\
\hline \multicolumn{15}{|l|}{ Occupation } \\
\hline Emploi à temps plein & 664 & 55,8 & 394 & 55,5 & 206 & 60,8 & 944 & 55,5 & 422 & 53,6 & 228 & 53,1 & 2858 & 55,4 \\
\hline Emploi à temps partiel & 80 & 6,7 & 59 & 8,3 & 17 & 5,0 & 109 & 6,4 & 58 & 7,4 & 41 & 9,6 & 364 & 7,1 \\
\hline Emploi autonome & 110 & 9,3 & 53 & 7,5 & 26 & 7,7 & 154 & 9,0 & 66 & 8,4 & 29 & 6,8 & 438 & 8,5 \\
\hline Sans emploi & 70 & 5,9 & 45 & 6,3 & 22 & 6,5 & 101 & 5,9 & 31 & 3,9 & 21 & 4,9 & 290 & 5,6 \\
\hline Études & 125 & 10,5 & 88 & 12,4 & 40 & 11,8 & 214 & 12,6 & 155 & 19,7 & 60 & 14,0 & 682 & 13,2 \\
\hline Retraite & 84 & 7,1 & 41 & 5,8 & 18 & 5,3 & 105 & 6,2 & 41 & 5,2 & 34 & 7,9 & 323 & 6,3 \\
\hline Autre & 56 & 4,7 & 30 & 4,2 & 10 & 2,9 & 75 & 4,4 & 14 & 1,8 & 16 & 3,7 & 201 & 3,9 \\
\hline \multicolumn{15}{|l|}{ Situation financière } \\
\hline Très confortable & 190 & 16,0 & 108 & 15,2 & 50 & 14,7 & 250 & 14,7 & 144 & 18,3 & 64 & 14,9 & 806 & 15,6 \\
\hline Confortable & 374 & 31,4 & 207 & 29,2 & 116 & 34,2 & 572 & 33,6 & 281 & 35,7 & 118 & 27,5 & 1668 & 32,3 \\
\hline Ni confortable ni inconfortable & 387 & 32,5 & 244 & 34,4 & 106 & 31,3 & 530 & 31,1 & 234 & 29,7 & 145 & 33,8 & 1646 & 31,9 \\
\hline Inconfortable & 170 & 14,3 & 102 & 14,4 & 42 & 12,4 & 235 & 13,8 & 105 & 13,3 & 76 & 17,7 & 730 & 14,2 \\
\hline Très inconfortable & 69 & 5,8 & 49 & 6,9 & 25 & 7,4 & 115 & 6,8 & 23 & 2,9 & 26 & 6,1 & 307 & 6,0 \\
\hline
\end{tabular}

de 36 ans. La majorité des participants ont déclaré être homosexuels $(76,0 \%)$ et le reste des participants, bisexuels $(17,5 \%)$, hétérosexuels $(0,9 \%)$ et un autre mot ou n'utilisant pas de mot (5,7\%). Parmi les participants, $2,4 \%$ ont déclaré être des hommes transgenres. En termes d'ethnicité, 3,8\% ont déclaré être autochtones, 3,8 \% latino-américains, 3,1\% asiatiques, $2,8 \%$ est-asiatiques ou sud-est-asiatiques, $1,9 \%$ sud-asiatiques, $1,7 \%$ noirs et $1,4 \%$ arabes/asiatiques de l'Ouest. Un cinquième $(20,7 \%)$ des participants était né hors du Canada.

Plus des trois quarts $(78,5 \%)$ des participants ont déclaré avoir suivi quatre années d'études ou plus après l'âge de 16 ans. Près de trois quarts $(71,0 \%)$ ont déclaré avoir un emploi (y compris un emploi à temps plein, à temps partiel ou autonome), alors que $13,2 \%$ ont déclaré être étudiants; le reste (15,8\%) a déclaré être sans emploi, retraité (y compris pour des raisons médicales), en congé de maladie à long terme ou autre. Près de la moitié $(47,9 \%)$ des participants a déclaré avoir une situation financière confortable; $31,9 \%$ ont déclaré ne vivre ni confortablement ni difficilement avec leurs revenus et un cinquième $(20,2 \%)$ a déclaré être dans une situation financière inconfortable.

\section{Expériences en matière de discrimination, de santé mentale et d'usage de substances}

Au cours de l'année précédant l'enquête, $31,9 \%$ des participants ont déclaré avoir fait l'objet d'intimidations, $22,1 \%$ ont été victimes de violence verbale et $1,5 \%$ de violence physique, parce que quelqu'un connaissait ou supposait cette attirance pour des hommes (tableau 2).

Près d'un quart $(23,9 \%)$ des participants a été classé dans les catégories modérée à aiguë de la mesure combinée d'anxiété et de dépression et $26,1 \%$ des participants ont signalé une idéation suicidaire allant d'occasionnelle à pratiquement quotidienne au cours des deux semaines précédentes.

En matière d'usage de substances, $64,1 \%$ ont déclaré avoir utilisé une substance illicite. L'usage de drogues le plus fréquemment déclaré a été celui du cannabis ( $46,6 \%)$, qui n'était pas encore légal au moment de l'enquête, de la cocainne $(14,0 \%)$ et de comprimés d'ecstasy ( $8,9 \%$ ) (tableau 2). Les substances utilisées au cours de l'année précédente par moins de $5 \%$ des participants globalement étaient les suivantes : amphétamines $(4,4 \%)$, kétamine $(4,0 \%)$, LSD $(3,9 \%)$, cannabinoïdes de synthèse $(2,1 \%)$, crack $(1,5 \%)$, héroïne $(0,9 \%)$, méphédrone $(0,4 \%)$ et stimulants de synthèse autres que la méphédrone $(0,5 \%)$. Seuls 3,5\% des participants ont déclaré avoir utilisé des drogues injectées. 
Tableau 2 : Expériences de discrimination relative à la connaissance ou à la présomption d'une attirance pour des hommes, santé mentale et usage de substances des participants canadiens à l'EMIS-2017 $(\mathrm{N}=5,165)$

\begin{tabular}{|c|c|c|c|c|c|c|c|c|c|c|c|c|c|c|}
\hline \multirow[t]{2}{*}{ Caractéristiques } & \multicolumn{2}{|c|}{$\begin{array}{c}\begin{array}{c}\text { Colombie- } \\
\text { Britannique } \\
\text { Yukon }\end{array} \\
(n=1191)^{\mathrm{a}}\end{array}$} & \multicolumn{2}{|c|}{$\begin{array}{c}\text { Alberta + } \\
\text { Territoires du } \\
\text { Nord-Ouest } \\
(n=710)^{\mathrm{a}}\end{array}$} & \multicolumn{2}{|c|}{$\begin{array}{c}\begin{array}{c}\text { Saskatchewan + } \\
\text { Manitoba }\end{array} \\
(n=339)^{\mathrm{a}}\end{array}$} & \multicolumn{2}{|c|}{$\begin{array}{c}\text { Ontario } \\
+ \\
\text { Nunavut } \\
(n=1707)^{a}\end{array}$} & \multicolumn{2}{|c|}{$\begin{array}{l}\text { Québec } \\
(\mathrm{n}=789)^{\mathrm{a}}\end{array}$} & \multicolumn{2}{|c|}{$\begin{array}{l}\begin{array}{c}\text { Provinces } \\
\text { atlantiques }\end{array} \\
(n=429)^{\mathrm{a}}\end{array}$} & \multicolumn{2}{|c|}{$\begin{array}{c}\text { Total } \\
(\mathrm{N}=5165)^{\mathrm{a}}\end{array}$} \\
\hline & $\mathbf{n}$ & $\%$ & $\mathbf{n}$ & $\%$ & n & $\%$ & $\mathbf{n}$ & $\%$ & $\mathbf{n}$ & $\%$ & $\mathbf{n}$ & $\%$ & $\mathbf{n}$ & $\%$ \\
\hline \multicolumn{15}{|c|}{ Dernière intimidation ou agression homophobe ${ }^{b}$} \\
\hline Au cours des six mois précédents & 172 & 14,5 & 94 & 13,3 & 43 & 12,7 & 233 & 13,7 & 67 & 8,5 & 38 & 8,9 & 647 & 12,6 \\
\hline Entre six mois et 1 an & 249 & 20,9 & 145 & 20,5 & 69 & 20,4 & 306 & 18,0 & 135 & 17,2 & 89 & 20,7 & 993 & 19,3 \\
\hline Entre 1 an et 5 ans & 190 & 16,0 & 96 & 13,5 & 36 & 10,6 & 240 & 14,1 & 115 & 14,6 & 60 & 14,0 & 737 & 14,3 \\
\hline Plus de 5 ans & 257 & 21,6 & 104 & 14,7 & 49 & 14,5 & 351 & 20,6 & 184 & 23,4 & 80 & 18,6 & 1025 & 19,9 \\
\hline Jamais & 321 & 27,0 & 270 & 38,1 & 142 & 41,9 & 570 & 33,5 & 286 & 36,3 & 162 & 37,8 & 1751 & 34,0 \\
\hline \multicolumn{15}{|l|}{ Insultes verbales ${ }^{b}$} \\
\hline Au cours des six mois précédents & 77 & 6,5 & 57 & 8,1 & 19 & 5,6 & 98 & 5,8 & 35 & 4,5 & 18 & 4,2 & 304 & 5,9 \\
\hline Entre six mois et 1 an & 223 & 18,8 & 113 & 16,0 & 62 & 18,3 & 289 & 17,0 & 83 & 10,6 & 64 & 15,0 & 834 & 16,2 \\
\hline Entre 1 an et 5 ans & 265 & 22,3 & 117 & 16,5 & 60 & 17,7 & 332 & 19,5 & 149 & 19,0 & 73 & 17,1 & 996 & 19,4 \\
\hline Plus de 5 ans & 323 & 27,2 & 137 & 19,4 & 70 & 20,6 & 460 & 27,1 & 219 & 27,9 & 122 & 28,5 & 1331 & 25,9 \\
\hline Jamais & 301 & 25,3 & 283 & 40,0 & 128 & 37,8 & 520 & 30,6 & 299 & 38,1 & 151 & 35,3 & 1682 & 32,7 \\
\hline \multicolumn{15}{|l|}{ Avoir été bousculé, frappé ou battub } \\
\hline Au cours des six mois précédents & 4 & 0,3 & 8 & 1,1 & 1 & 0,3 & 4 & 0,2 & 1 & 0,1 & 4 & 0,9 & 22 & 0,4 \\
\hline Entre six mois et 1 an & 17 & 1,4 & 10 & 1,4 & 5 & 1,5 & 11 & 0,6 & 7 & 0,9 & 6 & 1,4 & 56 & 1,1 \\
\hline Entre 1 an et 5 ans & 40 & 3,4 & 40 & 5,6 & 13 & 3,8 & 50 & 2,9 & 23 & 2,9 & 13 & 3,0 & 179 & 3,5 \\
\hline Plus de 5 ans & 216 & 18,2 & 83 & 11,7 & 34 & 10,0 & 292 & 17,2 & 125 & 15,9 & 71 & 16,6 & 821 & 15,9 \\
\hline Jamais & 913 & 76,7 & 568 & 80,1 & 286 & 84,4 & 1342 & 79,0 & 630 & 80,2 & 334 & 78,0 & 4073 & 79,1 \\
\hline \multicolumn{15}{|l|}{ Échelle d'anxiété et de dépression ${ }^{c}$} \\
\hline Normale & 472 & 40,0 & 276 & 39,1 & 137 & 40,7 & 731 & 43,4 & 321 & 41,3 & 188 & 44,7 & 2125 & 41,6 \\
\hline Moyenne & 393 & 33,3 & 249 & 35,3 & 116 & 34,4 & 546 & 32,4 & 308 & 39,6 & 149 & 35,4 & 1761 & 34,5 \\
\hline Modérée & 176 & 14,9 & 92 & 13,0 & 41 & 12,2 & 245 & 14,5 & 87 & 11,2 & 44 & 10,5 & 685 & 13,4 \\
\hline Aiguë & 139 & 11,8 & 88 & 12,5 & 43 & 12,8 & 164 & 9,7 & 61 & 7,9 & 40 & 9,5 & 535 & 10,5 \\
\hline \multicolumn{15}{|c|}{ Idéation suicidaire au cours des deux semaines précédentes } \\
\hline Pas du tout & 885 & 74,4 & 510 & 71,8 & 237 & 70,1 & 1268 & 74,7 & 592 & 75,2 & 311 & 72,5 & 3803 & 73,8 \\
\hline Certains jours & 200 & 16,8 & 129 & 18,2 & 70 & 20,7 & 305 & 18,0 & 148 & 18,8 & 87 & 20,3 & 939 & 18,2 \\
\hline Plus de la moitié des jours & 51 & 4,3 & 33 & 4,6 & 10 & 3,0 & 63 & 3,7 & 20 & 2,5 & 16 & 3,7 & 193 & 3,7 \\
\hline Presque chaque jour & 54 & 4,5 & 38 & 5,4 & 21 & 6,2 & 62 & 3,7 & 27 & 3,4 & 15 & 3,5 & 217 & 4,2 \\
\hline \multicolumn{15}{|l|}{ Dépendance à l'alcoold } \\
\hline Pas de dépendance à l'alcool & 936 & 79,1 & 553 & 78,4 & 262 & 77,7 & 1345 & 79,7 & 629 & 80,5 & 363 & 85,6 & 4088 & 79,9 \\
\hline Dépendance à l'alcool & 248 & 20,9 & 152 & 21,6 & 75 & 22,3 & 343 & 20,3 & 152 & 19,5 & 61 & 14,4 & 1031 & 20,1 \\
\hline \multicolumn{15}{|c|}{ Prise de drogues récréatives ou illicites } \\
\hline Non & 367 & 31,1 & 254 & 35,9 & 151 & 44,7 & 597 & 35,3 & 294 & 37,4 & 176 & 41,6 & 1839 & 35,9 \\
\hline Oui & 812 & 68,9 & 454 & 64,1 & 187 & 55,3 & 1092 & 64,7 & 492 & 62,6 & 247 & 58,4 & 3284 & 64,1 \\
\hline \multicolumn{15}{|c|}{ Drogues illicites prises au cours de l'année précédente ${ }^{e}$} \\
\hline Cannabis & 609 & 51,7 & 326 & 46,0 & 131 & 38,8 & 797 & 47,2 & 333 & 42,5 & 192 & 45,4 & 2388 & 46,6 \\
\hline Cocaïne & 192 & 16,3 & 98 & 13,9 & 39 & 11,5 & 222 & 13,2 & 123 & 15,6 & 40 & 9,5 & 714 & 14 \\
\hline Comprimés d'ecstasy & 152 & 12,9 & 53 & 7,5 & 15 & 4,4 & 147 & 8,7 & 77 & 9,5 & 16 & 3,8 & 458 & 8,9 \\
\hline $\begin{array}{l}\text { Ecstasy sous forme de poudre ou } \\
\text { crystal }\end{array}$ & 146 & 12,4 & 65 & 9,2 & 19 & 5,6 & 132 & 7,8 & 59 & 7,5 & 23 & 5,5 & 444 & 8,7 \\
\hline GHB ou GBL & 136 & 11,5 & 46 & 6,5 & 9 & 2,7 & 119 & 7,1 & 58 & 7,4 & 8 & 1,9 & 373 & 7,3 \\
\hline Crystal méthamphétamine & 80 & 6,8 & 43 & 6,1 & 16 & 4,7 & 129 & 7,6 & 29 & 3,7 & 13 & 3,1 & 310 & 6,1 \\
\hline \multicolumn{15}{|l|}{ Injection de drogues ${ }^{f}$} \\
\hline Jamais & 1139 & 96,0 & 679 & 95,8 & 328 & 96,8 & 1640 & 96,6 & 770 & 98,1 & 413 & 96,3 & 4969 & 96,5 \\
\hline Oui & 48 & 4,0 & 30 & 4,2 & 11 & 3,2 & 58 & 3,4 & 15 & 1,9 & 16 & 3,7 & 178 & 3,5 \\
\hline
\end{tabular}

Abréviations : GBL, gamma-butyrolactone; GHB, acide gamma-hydroxybutyrique

Les totaux pour chaque caractéristique n'atteignent pas toujours les totaux provinciaux, car seuls les participants pour qui aucune valeur ne manquait ont été inclus. Les valeurs manquantes représentent moins de $3 \%$ des totaux

b Parce que quelqu'un savait ou supposait que le participant était attiré par les hommes

'Mesure du Questionnaire sur la santé du patient-4 (13)

'Indicateur du questionnaire CAGE à 4 questions (14)

e Les dénominateurs des proportions de participants ayant utilisé chaque substance se fondent sur le nombre de participants ayant répondu à chaque question individuelle relative à l'usage de

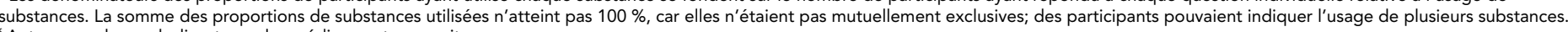

${ }^{\ddagger}$ Autres que des anabolisants ou des médicaments prescrits 


\section{Connaissance et utilisation de la prophylaxie postexposition (PEP) et préexposition (PrEP)}

La plupart des participants avaient entendu parler de la PrEP $(86,4 \%)$; une proportion légèrement inférieure avait entendu parler de la PEP (74,3\%) et $8,4 \%$ de tous les participants, quel que soit leur statut relatif au $\mathrm{VIH}$, avaient pris ou prenaient la PrEP (tableau 3). Parmi les hommes séronégatifs au VIH ou n'ayant pas fait de dépistage, $51,7 \%$ ont déclaré qu'ils utiliseraient probablement la PrEP si elle était disponible et abordable.

\section{Relations sexuelles}

Plus de la moitié des participants (62,1\%) ont toujours ou presque toujours eu des relations sexuelles sobres au cours des 12 mois précédents (tableau 4). Un cinquième (21,5\%) des participants a déclaré s'être adonné au chemsex et $5,8 \%$ ont déclaré l'avoir fait au cours des six mois précédents.

En matière de relations sexuelles, $62,1 \%$ des participants ont déclaré des pénétrations anales avec des partenaires occasionnels; parmi ces hommes, 73,6 \% n'avaient pas toujours utilisé de préservatifs au cours de l'année précédente (allant d'un à plusieurs rapport(s) sans préservatif).

Tableau 3 : Connaissance et utilisation de la PEP et de la PrEP et connaissance par professionnel de la santé au courant de l'attrait sexuel pour les hommes des participants canadiens à l'EMIS-2017 $(N=5,165)$

\begin{tabular}{|c|c|c|c|c|c|c|c|c|c|c|c|c|c|c|}
\hline \multirow[t]{2}{*}{ Caractéristiques } & \multicolumn{2}{|c|}{$\begin{array}{c}\text { Colombie- } \\
\text { Britannique + } \\
\text { Yukon } \\
(n=1191)^{\mathrm{a}}\end{array}$} & \multicolumn{2}{|c|}{$\begin{array}{l}\text { Alberta + } \\
\text { Territoires du } \\
\text { Nord-Ouest } \\
(n=710)^{a}\end{array}$} & \multicolumn{2}{|c|}{$\begin{array}{c}\text { Saskatchewan } \\
+ \\
\text { Manitoba } \\
(\mathrm{n}=339)^{\mathrm{a}}\end{array}$} & \multicolumn{2}{|c|}{$\begin{array}{c}\text { Ontario } \\
+ \\
\text { Nunavut } \\
(\mathrm{n}=1707)^{\mathrm{a}}\end{array}$} & \multicolumn{2}{|c|}{$\begin{array}{l}\text { Québec } \\
(\mathrm{n}=789)^{\mathrm{a}}\end{array}$} & \multicolumn{2}{|c|}{$\begin{array}{c}\text { Provinces } \\
\text { atlantiques } \\
(n=429)^{a}\end{array}$} & \multicolumn{2}{|c|}{$\begin{array}{c}\text { Total } \\
(\mathrm{N}=5165)^{\mathrm{a}}\end{array}$} \\
\hline & $n$ & $\%$ & $n$ & $\%$ & $n$ & $\%$ & $n$ & $\%$ & $n$ & $\%$ & $\mathbf{n}$ & $\%$ & $n$ & $\%$ \\
\hline \multicolumn{15}{|l|}{ Entendu parler de la PEP } \\
\hline Non & 157 & 13,4 & 175 & 24,8 & 76 & 22,6 & 265 & 15,7 & 167 & 21,3 & 117 & 27,8 & 957 & 18,7 \\
\hline Oui & 958 & 81,5 & 465 & 65,9 & 222 & 66,1 & 1323 & 78,6 & 560 & 71,5 & 268 & 63,7 & 3796 & 74,3 \\
\hline Pas certain & 61 & 5,2 & 66 & 9,3 & 38 & 11,3 & 96 & 5,7 & 56 & 7,2 & 36 & 8,6 & 353 & 6,9 \\
\hline \multicolumn{15}{|l|}{ Pris la PEPb } \\
\hline Non, jamais essayé de l'obtenir & 923 & 89,4 & 634 & 95,5 & 304 & 94,4 & 1395 & 91,8 & 629 & 87,1 & 386 & 95,5 & 4271 & 91,6 \\
\hline Non, tenté mais pas pu l'obtenir & 20 & 1,9 & 12 & 1,8 & 8 & 2,5 & 29 & 1,9 & 14 & 1,9 & 9 & 2,2 & 92 & 2,0 \\
\hline $\begin{array}{l}\text { Non, a eu l'occasion, mais a décidé } \\
\text { de ne pas la prendre }\end{array}$ & 14 & 1,4 & 5 & 0,8 & 4 & 1,2 & 19 & 1,3 & 13 & 1,8 & 1 & 0,2 & 56 & 1,2 \\
\hline Oui, plusieurs fois & 76 & 7,4 & 13 & 2,0 & 6 & 1,9 & 77 & 5,1 & 66 & 9,1 & 8 & 2,0 & 246 & 5,3 \\
\hline \multicolumn{15}{|l|}{ Certitude quant à l'accès à la PEPb } \\
\hline Très confiant & 322 & 31,2 & 145 & 21,9 & 71 & 22,0 & 470 & 30,9 & 262 & 36,2 & 69 & 17,0 & 1339 & 28,7 \\
\hline Plutôt confiant & 268 & 25,9 & 149 & 22,5 & 66 & 20,5 & 344 & 22,6 & 231 & 31,9 & 70 & 17,2 & 1128 & 24,1 \\
\hline Peu confiant & 173 & 16,7 & 126 & 19,0 & 64 & 19,9 & 287 & 18,8 & 98 & 13,5 & 93 & 22,9 & 841 & 18,0 \\
\hline Pas confiant du tout & 118 & 11,4 & 107 & 16,1 & 56 & 17,4 & 192 & 12,6 & 49 & 6,8 & 79 & 19,5 & 601 & 12,9 \\
\hline Ne sait pas & 152 & 14,7 & 136 & 20,5 & 65 & 20,2 & 230 & 15,1 & 84 & 11,6 & 95 & 23,4 & 762 & 16,3 \\
\hline \multicolumn{15}{|l|}{ A entendu parler de la PrEP } \\
\hline Non & 88 & 7,5 & 96 & 13,7 & 53 & 15,8 & 146 & 8,7 & 93 & 11,9 & 79 & 18,8 & 555 & 10,9 \\
\hline Oui & 1063 & 90,7 & 576 & 82,1 & 265 & 79,1 & 1506 & 89,3 & 669 & 85,7 & 326 & 77,6 & 4405 & 86,4 \\
\hline Pas certain & 21 & 1,8 & 30 & 4,3 & 17 & 5,1 & 34 & 2,0 & 19 & 2,4 & 15 & 3,6 & 136 & 2,7 \\
\hline \multicolumn{15}{|l|}{ Jamais pris la PrEP } \\
\hline Non & 1099 & 92,4 & 674 & 95,5 & 325 & 95,9 & 1532 & 90,3 & 664 & 84,7 & 415 & 97,6 & 4701 & 91,6 \\
\hline Oui & 90 & 7,6 & 32 & 4,6 & 14 & 4,1 & 165 & 9,7 & 120 & 15,3 & 10 & 2,4 & 431 & 8,4 \\
\hline \multicolumn{15}{|c|}{ Probabilité d'utiliser la PrEP si elle était disponible et abordable ${ }^{c}$} \\
\hline Très improbable & 117 & 11,3 & 46 & 6,9 & 30 & 9,3 & 143 & 9,4 & 88 & 12,2 & 36 & 8,9 & 460 & 9,9 \\
\hline Plutôt improbable & 111 & 10,7 & 50 & 7,6 & 39 & 12,1 & 134 & 8,8 & 99 & 13,7 & 32 & 7,9 & 465 & 10,0 \\
\hline Pas certain & 277 & 26,8 & 185 & 27,9 & 79 & 24,5 & 440 & 29,0 & 200 & 27,7 & 143 & 35,5 & 1324 & 28,4 \\
\hline Plutôt probable & 185 & 17,9 & 156 & 23,6 & 74 & 23,0 & 319 & 21,0 & 145 & 20,1 & 88 & 21,8 & 967 & 20,7 \\
\hline Très probable & 343 & 33,2 & 225 & 34,0 & 100 & 31,1 & 483 & 31,8 & 190 & 26,3 & 104 & 25,8 & 1445 & 31,0 \\
\hline
\end{tabular}

Abréviations : PEP, prophylaxie postexposition; PrEP, prophylaxie préexposition

a Les totaux pour chaque caractéristique n'atteignent pas toujours les totaux provinciaux, car seuls les participants pour qui aucune valeur ne manquait ont été inclus. Les valeurs manquantes représentent moins de $3 \%$ des totaux.

b Parmi tous les hommes séronégatifs au VIH ou n'ayant pas fait de dépistage $(\mathrm{N}=4665)$

'Parmi tous les hommes séronégatifs au $\mathrm{VIH}$ ou n'ayant pas fait de dépistage $(\mathrm{N}=4676)$ 
Tableau 4 : Relations sexuelles des participants canadiens à l'EMIS-2017 $(N=5,165)$

\begin{tabular}{|c|c|c|c|c|c|c|c|c|c|c|c|c|c|c|}
\hline \multirow[t]{2}{*}{ Caractéristiques } & \multicolumn{2}{|c|}{$\begin{array}{l}\text { Colombie- } \\
\text { Britannique + } \\
\text { Yukon } \\
(\mathrm{n}=1191)^{\mathrm{a}}\end{array}$} & \multicolumn{2}{|c|}{$\begin{array}{l}\text { Alberta + } \\
\text { Territoires du } \\
\text { Nord-Ouest } \\
(n=710)^{a}\end{array}$} & \multicolumn{2}{|c|}{$\begin{array}{c}\text { Saskatchewan }+ \\
\text { Manitoba } \\
(n=339)^{\mathrm{a}} \\
\end{array}$} & \multicolumn{2}{|c|}{$\begin{array}{c}\text { Ontario } \\
+ \\
\text { Nunavut } \\
(n=1707)^{\mathrm{a}} \\
\end{array}$} & \multicolumn{2}{|c|}{$\begin{array}{l}\text { Québec } \\
(\mathrm{n}=789)^{\mathrm{a}}\end{array}$} & \multicolumn{2}{|c|}{$\begin{array}{l}\text { Provinces } \\
\text { atlantiques } \\
(\mathrm{n}=429)^{\mathrm{a}}\end{array}$} & \multicolumn{2}{|c|}{$\begin{array}{c}\text { Total } \\
(\mathrm{N}=5165)^{\mathrm{a}}\end{array}$} \\
\hline & n & $\%$ & $\mathbf{n}$ & $\%$ & $\mathbf{n}$ & $\%$ & $\mathbf{n}$ & $\%$ & $\mathrm{n}$ & $\%$ & $\mathrm{n}$ & $\%$ & $\mathrm{n}$ & $\%$ \\
\hline \multicolumn{15}{|c|}{ Relations sexuelles non sobres (au cours des 12 mois précédents) ${ }^{b}$} \\
\hline Aucune & 362 & 33,1 & 252 & 41,0 & 125 & 41,7 & 533 & 35,0 & 253 & 35,8 & 160 & 44,3 & 1685 & 36,6 \\
\hline Quasiment aucune & 278 & 25,4 & 174 & 28,3 & 81 & 27,0 & 380 & 25,0 & 171 & 24,2 & 87 & 24,1 & 1171 & 25,5 \\
\hline Moins de la moitié & 141 & 12,9 & 71 & 11,6 & 26 & 8,7 & 209 & 13,7 & 92 & 13,0 & 42 & 11,6 & 581 & 12,6 \\
\hline La moitié environ & 115 & 10,5 & 42 & 6,8 & 21 & 7,0 & 122 & 8,0 & 67 & 9,5 & 24 & 6,6 & 391 & 8,5 \\
\hline Plus de la moitié & 74 & 6,8 & 27 & 4,4 & 9 & 3,0 & 111 & 7,3 & 49 & 6,9 & 16 & 4,4 & 286 & 6,2 \\
\hline \begin{tabular}{|l|} 
Presque toutes \\
\end{tabular} & 97 & 8,9 & 31 & 5,0 & 31 & 10,3 & 121 & 8,0 & 58 & 8,2 & 23 & 6,4 & 361 & 7,8 \\
\hline Toutes & 28 & 2,6 & 17 & 2,8 & 7 & 2,3 & 46 & 3,0 & 17 & 2,4 & 9 & 2,5 & 124 & 2,7 \\
\hline \multicolumn{15}{|l|}{ Récence du chemsex ${ }^{c}$} \\
\hline $\begin{array}{l}\text { Au cours des six mois } \\
\text { précédents }\end{array}$ & 95 & 8,1 & 26 & 3,7 & 11 & 3,3 & 105 & 6,2 & 47 & 6,0 & 14 & 3,3 & 298 & 5,8 \\
\hline Entre six mois et 1 an & 76 & 6,5 & 56 & 7,9 & 14 & 4,1 & 127 & 7,5 & 50 & 6,4 & 20 & 4,7 & 343 & 6,7 \\
\hline Entre 1 an et 5 ans & 50 & 4,2 & 37 & 5,2 & 10 & 3,0 & 62 & 3,7 & 31 & 3,9 & 12 & 2,8 & 202 & 3,9 \\
\hline Plus de 5 ans & 77 & 6,5 & 31 & 4,4 & 8 & 2,4 & 84 & 5,0 & 40 & 5,1 & 20 & 4,7 & 260 & 5,1 \\
\hline Jamais & 879 & 74,7 & 558 & 78,8 & 295 & 87,3 & 1311 & 77,6 & 617 & 78,6 & 357 & 84,4 & 4017 & 78,5 \\
\hline \multicolumn{15}{|c|}{ Pénétration anale avec des partenaires occasionnels (participants de tout statut VIH) } \\
\hline Aucune pénétration anale & 420 & 36 & 258 & 36,7 & 125 & 37,3 & 632 & 37,8 & 308 & 39,6 & 181 & 42,9 & 1924 & 37,9 \\
\hline $\begin{array}{l}\text { Tout type de pénétration } \\
\text { anale }\end{array}$ & 748 & 64 & 444 & 63,3 & 210 & 62,7 & 1038 & 62,2 & 470 & 60,4 & 241 & 57,1 & 3151 & 62,1 \\
\hline \multicolumn{15}{|c|}{ Utilisation systématique de préservatifs avec des partenaires occasionnels (participants de tout statut VIH) } \\
\hline $\begin{array}{l}\text { Utilisation non systématique } \\
\text { de préservatifs }\end{array}$ & 561 & 75,0 & 327 & 73,6 & 160 & 76,2 & 765 & 73,8 & 320 & 68,2 & 185 & 76,8 & 2318 & 73,6 \\
\hline $\begin{array}{l}\text { Utilisation systématique de } \\
\text { préservatifs }\end{array}$ & 187 & 25,0 & 117 & 26,4 & 50 & 23,8 & 272 & 26,2 & 149 & 31,8 & 56 & 23,2 & 831 & 26,4 \\
\hline \multicolumn{15}{|c|}{ PASP par des hommes séronégatifs au VIH utilisant la PrEPf } \\
\hline Non & - & - & - & - & - & - & - & - & - & - & - & - & 30 & 8,7 \\
\hline Oui & - & - & - & - & - & - & - & - & - & - & - & - & 314 & 91,3 \\
\hline \multicolumn{15}{|c|}{ PASP par des hommes séronégatifs au VIH ou qui ne connaissaient pas leur état de VIH et ne prenaient pas la PrEPg } \\
\hline Non & 180 & 26,5 & 114 & 27,4 & 50 & 25,1 & 265 & 29,5 & 134 & 36,1 & 54 & 23,4 & 797 & 28,5 \\
\hline Oui & 498 & 73,5 & 302 & 72,6 & 149 & 74,9 & 632 & 70,5 & 237 & 63,9 & 177 & 76,6 & 1995 & 71,5 \\
\hline
\end{tabular}

PASP par des hommes séropositifs au VIH sous traitement ayant une charge virale indétectable

\begin{tabular}{|c|c|c|c|c|c|c|c|c|c|c|c|c|c|c|}
\hline Non & - & - & - & - & - & - & - & - & - & - & - & - & 22 & 7,3 \\
\hline Oui & - & - & - & - & - & - & - & - & - & - & - & - & 278 & 92,7 \\
\hline \multicolumn{15}{|c|}{ PASP par des hommes séropositifs au VIH ne suivant pas de traitement et ayant une charge virale détectable } \\
\hline Non & - & - & - & - & - & - & - & - & - & - & - & - & 12 & 85,7 \\
\hline Oui & - & - & - & - & - & - & - & - & - & - & - & - & 2 & 14,3 \\
\hline \multicolumn{15}{|c|}{ A payé un homme pour des relations sexuelles } \\
\hline $\begin{array}{l}\text { Au cours des } 12 \text { mois } \\
\text { précédents }\end{array}$ & 57 & 4,9 & 33 & 4,8 & 24 & 7,4 & 108 & 6,6 & 51 & 6,8 & 14 & 3,5 & 287 & 5,8 \\
\hline Entre 1 an et 5 ans & 61 & 5,3 & 27 & 4,0 & 15 & 4,6 & 89 & 5,4 & 34 & 4,5 & 16 & 4,0 & 242 & 4,9 \\
\hline Plus de 5 ans & 78 & 6,7 & 31 & 4,6 & 19 & 5,9 & 113 & 6,9 & 38 & 5,1 & 18 & 4,4 & 297 & 6,0 \\
\hline Jamais & 961 & 83,1 & 590 & 86,6 & 266 & 82,1 & 1333 & 81,1 & 626 & 83,6 & 357 & 88,1 & 4133 & 83,3 \\
\hline \multicolumn{15}{|c|}{ A été payé par un homme pour des relations sexuelles } \\
\hline $\begin{array}{l}\text { Au cours des } 12 \text { mois } \\
\text { précédents }\end{array}$ & 40 & 3,5 & 30 & 4,4 & 7 & 2,2 & 67 & 4,1 & 36 & 4,8 & 20 & 4,9 & 200 & 4,0 \\
\hline Entre 1 an et 5 ans & 44 & 3,8 & 22 & 3,2 & 12 & 3,7 & 72 & 4,4 & 44 & 5,9 & 17 & 4,2 & 211 & 4,3 \\
\hline Plus de 5 ans & 114 & 9,9 & 43 & 6,3 & 24 & 7,4 & 153 & 9,3 & 33 & 4,4 & 25 & 6,2 & 392 & 7,9 \\
\hline Jamais & 959 & 82,9 & 586 & 86,0 & 281 & 86,7 & 1353 & 82,2 & 637 & 84,9 & 344 & 84,7 & 4160 & 83,8 \\
\hline
\end{tabular}

Abréviations : PASP, pénétration anale sans préservatif; PrEP, prophylaxie préexposition; - , nombre supprimé du fait d'une taille de cellule réduite

a Les totaux pour chaque caractéristique n'atteignent pas toujours les totaux provinciaux, car seuls les participants pour qui aucune valeur ne manquait ont été inclus. Les valeurs manquantes représentent moins de $5 \%$ des totaux

Parmi les hommes ayant eu des relations sexuelles avec un homme au cours des 12 mois précédents $(N=4599)$

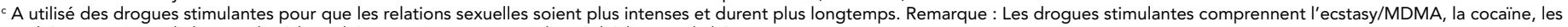
amphétamines (speed), la crystal méthamphétamine (Tina, Pervitin), la méphédrone et la kétamine

d Parmi les hommes de tout état relatif au VIH ayant eu un ou plusieurs partenaire(s) occasionnel(s) au cours des 12 mois précédents $(\mathrm{N}=5075)$

e Parmi les hommes de tout état relatif au VIH ayant participé à une pénétration anale avec un ou plusieurs partenaire(s) occasionnel(s) au cours des 12 mois précédents $(\mathrm{N}=3149)$

${ }^{\dagger}$ Parmi les hommes séronégatifs au VIH prenant la PrEP et ayant participé à une pénétration anale avec un ou plusieurs partenaire $(\mathrm{s})$ occasionnel(s) au cours des 12 mois précédents $(\mathrm{N}=344)$

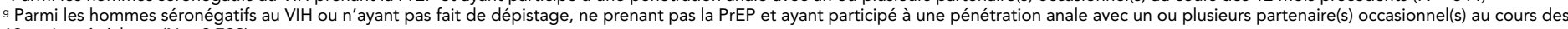

12 mois précédents $(\mathrm{N}=2792)$

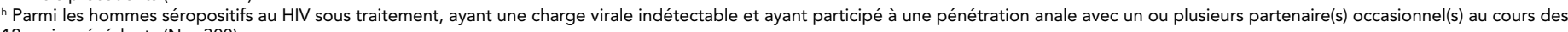

12 mois précédents $(\mathrm{N}=300)$

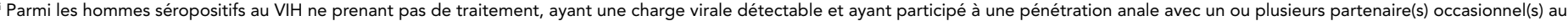
cours des 12 mois précédents $(\mathrm{N}=21)$ 
Parmi les hommes séronégatifs au VIH utilisant la PrEP et ayant participé à une pénétration anale avec un ou plusieurs partenaire(s) occasionnel(s) au cours de l'année précédente ( $n=344), 91,3 \%$ d'entre eux avaient participé à des pénétrations anales sans préservatif (PASP) avec des partenaires occasionnels au cours de l'année précédente. Parmi les hommes séronégatifs au VIH ou qui ne connaissaient pas leur état et ayant participé à une pénétration anale avec un ou plusieurs partenaire(s) occasionnel(s) au cours de l'année précédente et qui n'utilisaient pas de $\operatorname{PrEP}(n=2792), 71,5 \%$ d'entre eux avaient participé à des PASP avec des partenaires occasionnels au cours de I'année précédente. Parmi les hommes séropositifs au VIH ayant participé à une pénétration anale avec un ou plusieurs partenaire(s) occasionnel(s) au cours de l'année précédente et ayant une charge virale indétectable $(n=300), 92,7 \%$ d'entre eux avaient participé à des PASP avec des partenaires occasionnels au cours de l'année précédente. Parmi les hommes séropositifs au $\mathrm{VIH}$ ayant participé à une pénétration anale avec un ou plusieurs partenaire(s) occasionnel(s) au cours de l'année précédente, ne prenant pas de thérapie antirétrovirale et ayant une charge virale détectable $(n=14), 14,3 \%$ d'entre eux avaient participé à des PASP avec des partenaires occasionnels au cours de l'année précédente.

Au cours de l'année précédente, 5,8 \% des participants avaient payé pour avoir des rapports sexuels et $4,0 \%$ avaient été payés pour avoir des rapports sexuels avec des partenaires masculins.

\section{Dépistage et diagnostic d'ITSS}

Même si les deux tiers des participants (62,5\%) avaient fait un dépistage du VIH l'année précédente, seul un tiers (24,9\%) avait signalé un dépistage complet d'ITS au cours des 12 mois précédents (tableau 5). Cette proportion était supérieure $(37,7 \%)$ pour les participants ayant participé à toute PASP au cours de l'année précédente.

La plupart des participants (90\%) n'ayant pas fait de dépistage d'ITS autre que l'infection à VIH au cours de l'année précédente ont déclaré que leur fournisseur de soins de santé savait qu'ils avaient des relations sexuelles avec d'autres hommes.

En matière d'infections transmissibles par le sang, 1,5\% des participants avait reçu auparavant un diagnostic d'hépatite $C$ et $9 \%$ ont déclaré un diagnostic d'infection au $\mathrm{VIH}$. Parmi ces derniers, 99,1\% des participants séropositifs ont déclaré utiliser actuellement une thérapie antirétrovirale; chez ces derniers, $96,7 \%$ ont signalé une charge virale indétectable. Pour les autres ITSS, 3,2 \% des participants avaient reçu un diagnostic de syphilis l'année précédente. Des proportions supérieures de gonorrhée et de chlamydies (y compris le lymphogranulome vénérien) ont été signalées; pour respectivement 7,1\% et 6,5\% des participants ayant été diagnostiqués l'année précédente.

\section{Tableau 5 : Infections transmissibles sexuellement et par le sang et diagnostics des participants canadiens à I’EMIS-2017 ( $N=5,165)$}

\begin{tabular}{|c|c|c|c|c|c|c|c|c|c|c|c|c|c|c|}
\hline \multirow[t]{2}{*}{ Caractéristiques } & \multicolumn{2}{|c|}{$\begin{array}{c}\text { Colombie- } \\
\text { Britannique + } \\
\text { Yukon } \\
(n=1191)^{a}\end{array}$} & \multicolumn{2}{|c|}{$\begin{array}{c}\text { Alberta + } \\
\text { Territoires du } \\
\text { Nord-Ouest } \\
(n=710)^{a}\end{array}$} & \multicolumn{2}{|c|}{$\begin{array}{c}\text { Saskatchewan } \\
+ \\
\text { Manitoba } \\
(n=339)^{a}\end{array}$} & \multicolumn{2}{|c|}{$\begin{array}{c}\text { Ontario } \\
+ \\
\text { Nunavut } \\
(\mathrm{n}=1707)^{\mathrm{a}}\end{array}$} & \multicolumn{2}{|c|}{$\begin{array}{l}\text { Québec } \\
(n=789)^{a}\end{array}$} & \multicolumn{2}{|c|}{$\begin{array}{l}\text { Provinces } \\
\text { atlantiques } \\
(n=429)^{a}\end{array}$} & \multicolumn{2}{|c|}{$\begin{array}{c}\text { Total } \\
(\mathrm{N}=5165)^{\mathrm{a}}\end{array}$} \\
\hline & $\mathbf{n}$ & $\%$ & $\mathbf{n}$ & $\%$ & $\mathbf{n}$ & $\%$ & $\mathbf{n}$ & $\%$ & $\mathbf{n}$ & $\%$ & $\mathbf{n}$ & $\%$ & $\mathbf{n}$ & $\%$ \\
\hline \multicolumn{15}{|c|}{ Dépistage complet d'ITS au cours des 12 mois précédents ${ }^{b}$} \\
\hline Non & 698 & 66,7 & 480 & 71,6 & 246 & 76,4 & 1270 & 82,5 & 473 & 64,9 & 377 & 92,0 & 3544 & 75,1 \\
\hline Oui & 348 & 33,3 & 190 & 28,4 & 76 & 23,6 & 269 & 17,5 & 256 & 35,1 & 33 & 8,0 & 1172 & 24,9 \\
\hline \multicolumn{15}{|c|}{ Personnel de santé au courant des relations sexuelles avec d'autres hommes ${ }^{c}$} \\
\hline Définitivement/Probablement & 718 & 91,8 & 358 & 86,1 & 153 & 86,4 & 883 & 91,3 & 451 & 93,8 & 155 & 78,7 & 2718 & 90,0 \\
\hline Pas au courant ou ne sait pas & 64 & 8,2 & 58 & 13,9 & 24 & 13,6 & 84 & 8,7 & 30 & 6,2 & 42 & 21,3 & 302 & 10,0 \\
\hline \multicolumn{15}{|l|}{ Historique de dépistage du VIH ${ }^{d}$} \\
\hline Au cours des six mois précédents & 213 & 20,6 & 94 & 14,2 & 39 & 12,1 & 224 & 14,7 & 131 & 18,1 & 26 & 6,4 & 727 & 15,5 \\
\hline Entre six mois et 1 an & 507 & 49,0 & 311 & 46,8 & 150 & 46,6 & 731 & 47,9 & 327 & 45,2 & 173 & 42,6 & 2199 & 47,0 \\
\hline Entre 1 an et 5 ans & 151 & 14,6 & 91 & 13,7 & 36 & 11,2 & 219 & 14,4 & 103 & 14,2 & 67 & 16,5 & 667 & 14,3 \\
\hline Plus de 5 ans & 45 & 4,4 & 26 & 3,9 & 22 & 6,8 & 83 & 5,4 & 28 & 3,9 & 25 & 6,2 & 229 & 4,9 \\
\hline Jamais & 118 & 11,4 & 142 & 21,4 & 75 & 23,3 & 269 & 17,6 & 135 & 18,6 & 115 & 28,3 & 854 & 18,3 \\
\hline \multicolumn{15}{|l|}{ Dernier diagnostic de syphilis } \\
\hline Au cours des 12 mois précédents & 50 & 4,2 & 21 & 3,0 & 8 & 2,4 & 53 & 3,2 & 25 & 3,2 & 5 & 1,2 & 162 & 3,2 \\
\hline Entre 1 an et 5 ans & 71 & 6,0 & 24 & 3,4 & 16 & 4,8 & 93 & 5,5 & 47 & 6,1 & 20 & 4,7 & 271 & 5,3 \\
\hline Plus de 5 ans & 44 & 3,7 & 22 & 3,1 & 9 & 2,7 & 84 & 5,0 & 19 & 2,5 & 14 & 3,3 & 192 & 3,8 \\
\hline Jamais & 1015 & 86,0 & 635 & 90,5 & 301 & 90,1 & 1447 & 86,3 & 684 & 88,3 & 387 & 90,8 & 4469 & 87,7 \\
\hline \multicolumn{15}{|l|}{ Dernier diagnostic de gonorrhée } \\
\hline Au cours des 12 mois précédents & 110 & 9,3 & 43 & 6,1 & 19 & 5,7 & 99 & 5,9 & 77 & 10,0 & 11 & 2,6 & 359 & 7,1 \\
\hline Entre 1 an et 5 ans & 147 & 12,5 & 60 & 8,5 & 22 & 6,6 & 135 & 8,0 & 72 & 9,3 & 17 & 4,0 & 453 & 8,9 \\
\hline Plus de 5 ans & 131 & 11,1 & 60 & 8,5 & 22 & 6,6 & 180 & 10,7 & 65 & 8,4 & 32 & 7,5 & 490 & 9,6 \\
\hline Jamais & 790 & 67,1 & 540 & 76,8 & 268 & 81,0 & 1268 & 75,4 & 559 & 72,3 & 365 & 85,9 & 3790 & 74,4 \\
\hline
\end{tabular}


Tableau 5 : Infections transmissibles sexuellement et par le sang et diagnostics des participants canadiens à I'EMIS-2017 ( $N=5,165)$ (suite)

\begin{tabular}{|c|c|c|c|c|c|c|c|c|c|c|c|c|c|c|}
\hline \multirow[t]{2}{*}{ Caractéristiques } & \multicolumn{2}{|c|}{$\begin{array}{c}\text { Colombie- } \\
\text { Britannique + } \\
\text { Yukon } \\
(n=1191)^{a}\end{array}$} & \multicolumn{2}{|c|}{$\begin{array}{c}\text { Alberta + } \\
\text { Territoires du } \\
\text { Nord-Ouest } \\
(n=710)^{a}\end{array}$} & \multicolumn{2}{|c|}{$\begin{array}{c}\text { Saskatchewan } \\
+ \\
\text { Manitoba } \\
(n=339)^{a}\end{array}$} & \multicolumn{2}{|c|}{$\begin{array}{c}\text { Ontario } \\
+ \\
\text { Nunavut } \\
(\mathrm{n}=1707)^{\mathrm{a}}\end{array}$} & \multicolumn{2}{|c|}{$\begin{array}{l}\text { Québec } \\
(n=789)^{a}\end{array}$} & \multicolumn{2}{|c|}{$\begin{array}{l}\text { Provinces } \\
\text { atlantiques } \\
(n=429)^{a}\end{array}$} & \multicolumn{2}{|c|}{$\begin{array}{c}\text { Total } \\
(\mathrm{N}=5165)^{\mathrm{a}}\end{array}$} \\
\hline & $\mathbf{n}$ & $\%$ & $\mathbf{n}$ & $\%$ & $\mathbf{n}$ & $\%$ & $\mathbf{n}$ & $\%$ & n & $\%$ & $\mathbf{n}$ & $\%$ & $\mathbf{n}$ & $\%$ \\
\hline \multicolumn{15}{|c|}{ Dernier diagnostic de chlamydies ou LGV } \\
\hline Au cours des 12 mois précédents & 105 & 9,0 & 35 & 5,1 & 14 & 4,3 & 106 & 6,4 & 60 & 7,8 & 7 & 1,7 & 327 & 6,5 \\
\hline Entre 1 an et 5 ans & 109 & 9,3 & 57 & 8,2 & 26 & 7,9 & 140 & 8,4 & 67 & 8,7 & 18 & 4,3 & 417 & 8,2 \\
\hline Plus de 5 ans & 94 & 8,0 & 43 & 6,2 & 24 & 7,3 & 127 & 7,6 & 42 & 5,4 & 21 & 5,0 & 351 & 6,9 \\
\hline Jamais & 865 & 73,7 & 558 & 80,5 & 265 & 80,5 & 1293 & 77,6 & 604 & 78,1 & 375 & 89,1 & 3960 & 78,3 \\
\hline \multicolumn{15}{|l|}{ Diagnostic d'hépatite C } \\
\hline Non & 1135 & 95,3 & 672 & 94,9 & 331 & 97,9 & 1648 & 96,8 & 765 & 97,2 & 408 & 95,8 & 4959 & 96,3 \\
\hline Oui & 26 & 2,2 & 13 & 1,8 & 0 & 0,0 & 23 & 1,4 & 11 & 1,4 & 3 & 0,7 & 76 & 1,5 \\
\hline Ne sait pas & 30 & 2,5 & 23 & 3,2 & 7 & 2,1 & 31 & 1,8 & 11 & 1,4 & 15 & 3,5 & 117 & 2,3 \\
\hline \multicolumn{15}{|l|}{ Diagnostic de VIH } \\
\hline Non & 1034 & 87,4 & 664 & 94,1 & 322 & 95,0 & 1526 & 89,8 & 724 & 92,0 & 406 & 95,5 & 4676 & 91,0 \\
\hline Oui & 149 & 12,6 & 42 & 5,9 & 17 & 5,0 & 174 & 10,2 & 63 & 8,0 & 19 & 4,5 & 464 & 9,0 \\
\hline \multicolumn{15}{|l|}{ Traitement ARV en cours } \\
\hline Non & - & - & - & - & - & - & - & - & - & - & - & - & 4 & 0,9 \\
\hline Oui & - & - & - & - & - & - & - & - & - & - & - & - & 436 & 99,1 \\
\hline \multicolumn{15}{|c|}{ Charge virale détectable au dernier suivif } \\
\hline Indétectable & - & - & - & - & - & - & - & - & - & - & - & - & 440 & 96,7 \\
\hline Détectable & - & - & - & - & - & - & - & - & - & - & - & - & 15 & 3,3 \\
\hline $\begin{array}{l}\text { Abréviations : ITS, infections transmissib } \\
\text { Les totaux pour chaque caractéristique } \\
\text { eprésentent moins de } 3 \% \text { des totaux } \\
\text { Dépistage complet d'ITS au cours des } \\
\text { une infection au VIH depuis plus d'un ar } \\
\text { Parmi les hommes ayant fait un dépist } \\
\text { Parmi tous les hommes séronégatifs a } \\
\text { Parmi les hommes ayant auto-déclaré } \\
\text { Parmi les hommes ayant auto-déclaré }\end{array}$ & $\begin{array}{l}\text { sexuellen } \\
\text { 'atteignen } \\
\text { mois pré } \\
\text { = } 4716 \text { ) } \\
\text { d'ITS aut } \\
\text { IH ou n'ay } \\
\text { diagnosti } \\
\text { diagnostic }\end{array}$ & $\begin{array}{l}\text { as toujou } \\
\text { ents : inf } \\
\text { que l'infe } \\
\text { pas fait } \\
\text { VIH et a }\end{array}$ & $\begin{array}{l}\text { mphogranu } \\
\mathrm{s} \text { les totaux } \\
\text { ction au VI } \\
\text { ction à VIH } \\
\text { le dépistag } \\
\text { yant répon }\end{array}$ & $\begin{array}{l}\text { vincia } \\
\text { nalyse } \\
\text { ours } \\
=46 \\
\text { la }\end{array}$ & $\begin{array}{l}\text { car seuls } \\
\text { e sang, pr } \\
\text { l'année pr }\end{array}$ & $\begin{array}{l}\text { particip } \\
\text { ement a } \\
\text { dente ( }\end{array}$ & $\begin{array}{l}\text { s pour qu } \\
\text { I et urétra } \\
\begin{array}{l}3020 \text { ) } \\
N=440 \text { ) } \\
J=455 \text { ) }\end{array}\end{array}$ & $\begin{array}{l}\text { ucune } \mathrm{v} \\
\text { u prélè }\end{array}$ & ent v & et ana & d'urir & exclua & réduit & ayant \\
\hline
\end{tabular}

\section{Discussion}

L'EMIS-2017 a relevé que les participants canadiens ont subi des niveaux élevés d'intimidation ainsi que de la violence verbale et physique du fait de leur attirance par d'autres hommes. Près de $25 \%$ des participants souffraient de formes aiguës d'anxiété ou de dépression. L'usage de substances était élevé et, pour la première fois, des données nationales indiquaient que plus de $20 \%$ des participants s'adonnaient au chemsex. II existe un écart significatif entre la proportion des participants ayant déclaré utiliser la PrEP et celle de participants déclarant qu'ils I'utiliseraient si elle était facilement accessible : 8,4 \% par rapport à $51,7 \%$. Même si pratiquement tous les répondants ayant reçu un diagnostic de $\mathrm{VIH}$ suivaient un traitement et avaient une charge virale indétectable, moins de $25 \%$ ont déclaré avoir fait l'objet d'un dépistage complet d'ITS au cours de l'année précédente.

\section{Forces et limites}

La force de l'enquête EMIS-2017 canadienne a été l'utilisation d'un questionnaire mondial validé facilitant les comparaisons à l'échelle du pays, sa taille d'échantillon élevée et la participation de toutes les régions canadiennes.
Plusieurs limites doivent être prises en compte lors de I'interprétation des résultats. Puisque l'EMIS-2017 se fonde sur des données auto-déclarées et porte sur des sujets délicats, comme les relations sexuelles et l'usage de substances, un certain degré de sous-déclaration de comportements à plus haut risque est possible. Cependant, toute sous-déclaration était probablement limitée du fait de la nature autoadministrée de l'enquête. L'EMIS-2017 a eu recours à des méthodes d'échantillonnage non probabiliste, notamment l'utilisation d'applications de réseaux sociaux et sexuels pour le recrutement; par conséquent, cette enquête représente plus probablement des hommes gbHARSAH non monogames sexuellement actifs. Cependant, il s'agit d'une des principales populations cibles pour les enquêtes de comportement et les interventions de santé publique.

\section{Répercussions}

Bon nombre des résultats reflètent ceux d'études antérieures. Une enquête canadienne sur les hommes gbHARSAH a, par exemple, relevé un risque plus élevé d'idéation suicidaire et de comportement associé chez les hommes gbHARSAH que chez les hommes hétérosexuels (17). Un examen de la littérature internationale a indiqué une prévalence supérieure d'usage de substances chez les hommes gbHARSAH que chez les hommes 
hétérosexuels (18). Le recours déclaré au chemsex (21,5\%) dans la portion canadienne de l'EMIS-2017 était supérieur aux résultats d'études canadiennes régionales (6\% et $18 \%)(19,20)$ et peut refléter une variation régionale. D'autres études ont également relevé que les défis en matière de santé mentale et d'usage de substances étaient associés au fait que les hommes gbHARSAH s'adonnaient à des pratiques sexuelles à risque plus élevé (21-23). La tendance de la coïncidence de ces problèmes a été conceptualisée comme étant syndémique; c.-à-d. des épidémies concomitantes entraînant une charge de morbidité plus élevée dans des populations marginalisées (24). Des études antérieures ont indiqué que dans certaines villes, entre 10,5\% et $12,5 \%$ des hommes gbHARSAH utilisaient la PrEP (25) et que de $50 \%$ à $60 \%$ des hommes gbHARSAH souhaitaient avoir recours à la PrEP $(26,27)$; ce qui suggère que l'abordabilité et I'accessibilité sont des obstacles $(28,29)$. Une faible proportion des participants déclarant un dépistage complet d'ITS a été également relevée dans d'autres enquêtes auprès d'hommes gbHARSAH au Canada (30) et au niveau international $(31,32)$. Le résultat indiquant que pratiquement tous les participants ayant reçu un diagnostic d'infection au $\mathrm{VIH}$ suivaient un traitement et avaient une charge virale indétectable est similaire à celui $d$ 'enquêtes régionales récentes menées auprès $d$ 'hommes gbHARSAH $(33,34)$.

\section{Étapes suivantes}

Les résultats de l'EMIS-2017 soulignent le besoin de mettre en œuvre des recherches permettant d'établir des pratiques exemplaires pour faire face aux niveaux élevés de dangers associés à la discrimination, à la piètre santé mentale et à l'usage de substances auxquels les hommes gbHARSAH font face. L'étude EMIS-2017 fournit également des données de base utiles sur la PrEP. Du fait de modifications apportées aux formulaires provinciaux et aux récentes lignes directrices sur la prescription de la PrEP, nous anticipons une plus grande adoption de cette technologie de prévention efficace. De plus amples recherches seraient utiles pour déterminer le rôle de la PrEP dans les pratiques sexuelles à risque et les taux ultérieurs d'infection au VIH et à d'autres ITSS. Puisque cette enquête capture probablement un sous-groupe particulier et important des hommes gbHARSAH quant aux ITSS, une triangulation de ces données avec des renseignements générés à partir d'enquêtes futures à l'aide d'autres méthodes d'échantillonnage mènerait à une compréhension plus complète de cette population dans son ensemble.

\section{Conclusion}

Les hommes gbHARSAH au Canada ont souffert d'un degré élevé de stigmatisation, de discrimination et de troubles mentaux, alors que l'usage de substances ainsi qu'une faible utilisation de préservatif ont été relevés. L'écart entre la proportion des hommes intéressés par la PrEP et ceux I'utilisant effectivement était significatif et le dépistage complet
d'ITSS était faible. Ces résultats peuvent aider les mesures de santé publique et fournir une base d'examen de l'incidence d'interventions actuelles et nouvelles.

\section{Déclaration des auteurs}

N. B. - Conceptualisation, organisation des données, analyse formelle, rédaction (ébauche initiale, révision et modification) D. M. P. - Acquisition du financement, administration du projet, conceptualisation, organisation des données, analyse formelle, rédaction, révision et modification

N. J. L. - Conceptualisation, analyse formelle, rédaction (révision et modification)

M. B. - Conceptualisation, analyse formelle, rédaction (révision et modification)

D. J. B. - Conceptualisation, analyse formelle, rédaction (révision et modification)

T. A. H. - Conceptualisation, analyse formelle, rédaction (révision et modification)

B. A. - Conceptualisation, analyse formelle, rédaction (révision et modification)

\section{Conflit d'intérêts}

Aucun.

\section{Remerciements}

L'Enquête européenne en ligne sur la sexualité entre hommes (EMIS-2017) a été menée par Sigma Research à la London School of Hygiene and Tropical Medicine en association avec l'Institut Robert-Koch de Berlin. L'équipe de base de l'EMIS est constituée de F. Hickson, D. Reid, A. J. Schmidt et P. Weatherburn de la London School of Hygiene and Tropical Medicine ainsi que d'U. Marcus et S. B. Schink de I'Institut Robert Koch. Nous remercions en particulier A. J. Schmidt pour son soutien solide et continu à la participation canadienne à l'EMIS-2017.

Nous remercions tous les hommes ayant pris part à I'EMIS-2017 et nos partenaires d'organismes non gouvernementaux (ONG), en particulier la Health Initiative for Men, Rézo, la Gay Men's Sexual Health Alliance de I'Ontario et CATIE (le Réseau canadien d'info-traitements sida), qui ont recruté les participants grâce à des activités sur Facebook et/ou d'autres réseaux sociaux et en affichant des bannières pour l'EMIS sur leurs sites Web. Nous remercions tous nos partenaires d'avoir pris part à cette importante initiative. Nous souhaitons également remercier C. Archibald pour ses conseils avant et tout au long de la collecte de données et pour sa révision du manuscrit. 


\section{Financement}

Dans le cadre du projet ESTICOM (enquêtes et formations européennes visant à améliorer la santé des communautés HARSAH), I'EMIS-2017 a été menée en vertu du contrat de service 20157101 avec la Chafea (organisme de direction en matière de consommation, de santé, d'agriculture et d'alimentation), agissant selon des pouvoirs délégués par la Commission européenne. D'autres contributions financières provenaient de l'Agence de la santé publique du Canada (ASPC) pour le recrutement au Canada. N. J. L. est soutenu par une bourse de recherche de la Michael Smith Foundation for Health Research (\#16863). T. A. H. est soutenu par une chaire Endgame Leader de I'Ontario HIV Treatment Network. La promotion de l'enquête et le recrutement des participants canadiens à I'EMIS-2017 ont été financés par l'ASPC.

\section{Références}

1. The ESTICOM Network. EMIS-2017 - The European MenWho-Have-Sex-With-Men Internet Survey. Key finding from 50 countries. Stockholmn (SE): European Centre for Disease Prevention and Control; 2019 (Accédé 2019-09-04). http://www.emis2017.eu

2. Public Health Agency of Canada. Summary: estimates of HIV incidence, prevalence and Canada's progress on meeting the 90-90-90 HIV targets, 2016. Ottawa (ON): PHAC; 2018. https://www.canada.ca/content/dam/phac-aspc/documents/ services/publications/diseases-conditions/summary-estimate s-hiv-incidence-prevalence-canadas-progress-90-90-90/ pub-eng.pdf

3. Choudhri Y, Miller J, Sandhu J, Leon A, Aho J. Infectious and congenital syphilis in Canada, 2010-2015. Can Commun Dis Rep 2018 Feb;44(2):43-8. DOI PubMed

4. Choudhri Y, Miller J, Sandhu J, Leon A, Aho J. Gonorrhea in Canada, 2010-2015. Can Commun Dis Rep 2018 Feb;44(2):37-42. DOI PubMed

5. Ling DI, Janjua NZ, Wong S, Krajden M, Hoang L, Morshed M, Achen M, Murti M, Lester RT, Wong J, Ogilvie G, Gilbert $M$. Sexually transmitted infection trends among gay or bi-sexual men from a clinic-based sentinel surveillance system in British Columbia, Canada. Sex Transm Dis 2015 Mar;42(3):153-9. DOI PubMed

6. Mohammed H, Mitchell H, Sile B, Duffell S, Nardone A, Hughes $G$. Increase in sexually transmitted infections among men who have sex with men, England, 2014. Emerg Infect Dis 2016 Jan;22(1):88-91. DOI PubMed

7. Rietmeijer CA, Patnaik JL, Judson FN, Douglas JM Jr. Increases in gonorrhea and sexual risk behaviors among men who have sex with men: a 12-year trend analysis at the Denver Metro Health Clinic. Sex Transm Dis 2003 Jul;30(7):562-7. DOI PubMed
8. Jordan AE, Perlman DC, Neurer J, Smith DJ, Des Jarlais DC, Hagan $\mathrm{H}$. Prevalence of hepa-titis $\mathrm{C}$ virus infection among $\mathrm{HIV}+$ men who have sex with men: a systematic review and meta-analysis. Int J STD AIDS 2017 Feb;28(2):145-59. DOI PubMed

9. Pakianathan M, Whittaker W, Lee MJ, Avery J, Green S, Nathan B, Hegazi A. Chemsex and new HIV diagnosis in gay, bisexual and other men who have sex with men attending sexual health clinics. HIV Med 2018 May;19(7):485-90. DOI PubMed

10. Institute of Medicine. The health of lesbian, gay, bisexual, and transgender people: build-ing a foundation for better understanding. Washington (DC): National Academies Press; 2011. https://www.ncbi.nlm.nih.gov/books/NBK64806/pdf/ Bookshelf_NBK64806.pdf

11. Weatherburn P, Hickson F, Reid DS, Marcus U, Schmidt AJ. European Men-who-have-sex-with-men Internet Survey (EMIS-2017): design and methods. Sex Res Soc Policy 2019 (in press).

12. The EMIS Network. EMIS-2017: 33 language versions of the European Men-who-have-sex-with-men Internet Survey. The EMIS Network; 2017 (Accédé 2019-09-04). http:// sigmaresearch.org.uk/tags/all/tag/EMIS-2017

13. Kroenke K, Spitzer RL, Williams JB, Löwe B. An ultra-brief screening scale for anxiety and depression: the PHQ-4. Psychosomatics 2009 Nov-Dec;50(6):613-21. DOl PubMed

14. Shields AL, Caruso JC. A reliability induction and reliability generalization study of the Cage Questionnaire. Educ Psychol Meas 2004;64(2):254-70. DOI

15. Holt M, Lea T, Mao L, Zablotska I, Lee E, de Wit JB, Prestage G. Adapting behavioural surveillance to antiretroviral-based HIV prevention: reviewing and anticipating trends in the Australian Gay Community Periodic Surveys. Sex Health 2017 Feb;14(1):72-9. DOI PubMed

16. European Centre for Disease Prevention and Control. Monitoring implementation of the Dublin Declaration. Solna (SE): ECDC. https://ecolc.europa.eu/en/all-topic s-zhiv-infection-and-aidsprevention-and-control/ monitoring-implementation-dublin

17. Ferlatte O, Dulai J, Hottes TS, Trussler T, Marchand R. Suicide related ideation and behav-ior among Canadian gay and bisexual men: a syndemic analysis. BMC Public Health 2015 Jul;15(1):597. DOl PubMed

18. Bourne A, Weatherburn P. Substance use among men who have sex with men: patterns, motivations, impacts and intervention development need. Sex Transm Infect 2017 Aug;93(5):342-6. DOl PubMed

19. Noor SW, Adam BD, Brennan DJ, Moskowitz DA, Gardner S, Hart TA. Scenes as micro-cultures: examining heterogeneity of HIV risk behavior among gay, bisexual, and other men who have sex with men in Toronto, Canada. Arch Sex Behav 2018 Jan;47(1):309-21. DOl PubMed 
20. Messier-Peet M, Apelian H, Lambert G, Cox J. Chemsex \& mental health in gbMSM in Montreal: results from Engage Cycle 1. Gay Men's Health Summit, 2018. Vancouver (BC); 2018 Nov 8-9. https://www.engage-men.ca/wp-content/ uploads/2018/04/Engage-Montr\%C3\%A9al-Chemse x-Mental-Health-FINAL-ENGLISH.pdf

21. Card KG, Lachowsky NJ, Armstrong HL, Cui Z, Wang L, Sereda P, Jollimore J, Patterson TL, Corneil T, Hogg RS, Roth EA, Moore DM. The additive effects of depressive symp-toms and polysubstance use on HIV risk among gay, bisexual, and other men who have sex with men. Addict Behav 2018 Jul;82:158-65. DOI PubMed

22. Grewal R, Allen VG, Gardner S, Moravan V, Tan DH, Raboud J, Bayoumi AM, Kaul R, Mazzulli T, McGee F, Rourke SB, Burchell AN; OHTN Cohort Study Research Team. Se-rosorting and recreational drug use are risk factors for diagnosis of genital infection with chlamydia and gonorrhoea among HIV-positive men who have sex with men: results from a clinical cohort in Ontario, Canada. Sex Transm Infect 2017 Feb;93(1):71-5. DOI PubMed

23. Hart TA, Noor SW, Adam BD, Vernon JR, Brennan DJ, Gardner S, Husbands W, Myers T. Correction: Number of psychosocial strengths predicts reduced HIV sexual risk behaviors above and beyond syndemic problems among gay and bisexual men. AIDS Behav 2017 Oct;21(10):3035-46. DOI

24. Batchelder AW, Safren S, Mitchell AD, Ivardic I, O'Cleirigh C. Mental health in 2020 for men who have sex with men in the United States. Sex Health 2017 Feb;14(1):59-71.

DOI PubMed

25. Armstrong $\mathrm{H}$, Pan S, Barath J, Olarewaju G, Rodrigues R, Messier-Preet M, Apelian H, Hull M, Tan D, Lachowsky N, Jollimore J, Hart T, Grace D, Lambert G, Cox J, Moore D. Trends in awareness and use of PrEP among HIV-negative men who have sex with men in Vancouver, Toronto, and Montreal. Sex Transm Infect 2019;95:A231-2. https://sti.bmj. com/content/sextrans/95/Suppl_1/A231.2.full.pdf

26. Morgan J, Ferlatte $O$, Salway T, Wilton J, Hull M. Awareness of, interest in, and willing-ness to pay for HIV pre-exposure prophylaxis among Canadian gay, bisexual, and other men who have sex with men. Can J Public Health 2018 Dec;109(56):791-9. DOI PubMed

27. Hall EW, Heneine W, Sanchez T, Sineath RC, Sullivan P. Preexposure prophylaxis modali-ty preferences among men who have sex with men and use social media in the United States. J Med Internet Res 2016 May;18(5):e111. DOI PubMed
28. Rapid Response Service. Facilitators and barriers to health care for lesbian, gay and bisex-ual (LGB) people. Toronto (ON): Ontario HIV Treatment Network; 2014 Mar. https://www.ohtn.on.ca/Pages/Knowledge-Exchange/ Rapid-Responses/Documents/RR79.pdf

29. Mosley T, Khaketla M, Armstrong HL, Cui Z, Sereda P, Lachowsky NJ, Hull MW, Olarewaju G, Jollimore J, Edward J, Montaner JS, Hogg RS, Roth EA, Moore DM. Trends in awareness and use of HIV PrEP among gay, bisexual, and other men who have sex with men in Vancouver, Canada 2012-2016. AIDS Behav 2018 Nov;22(11):3550-65. DOl PubMed

30. Ha S, Whyte CA, Paquette D, Tarasuk J, Latham-Carmanico C, Holt M. Comprehensive testing for sexually transmitted and blood-borne infections among men who have sex with men in Canada: findings from an enhanced surveillance system (M-Track 2005-2007). J Infect Dis 2014;25 Suppl 4:75A. https://www.cahr-acrv.ca/ wp-content/uploads/2012/10/CAHR-2014-Abstract-Book.pdf

31. Frankis J, Goodall L, Clutterbuck D, Abdul-Razak A, Flowers P. Regular STI testing amongst men who have sex with men and use social media is suboptimal - a cross-sectional study. Int J STD AIDS 2017 May;28(6):573-83. DOI PubMed

32. Holt M, Hull P, Lea T, Guy R, Bourne C, Prestage G, Zablotska I, de Wit J, Mao L. Com-prehensive testing for, and diagnosis of, sexually transmissible infections among Australi-an gay and bisexual men: findings from repeated, cross-sectional behavioural surveillance, 2003-2012. Sex Transm Infect 2014 May;90(3):208-15. DOI PubMed

33. Lourenço L, Colley G, Nosyk B, Shopin D, Montaner JS, Lima VD; STOP HIV/AIDS Study Group. High levels of heterogeneity in the HIV cascade of care across different population subgroups in British Columbia, Canada. PLoS One 2014 Dec;9(12):e115277. DOI PubMed

34. Lambert G, Cox J, Messier-Peet M, Apelian H, Moodie EE. Engage Montréal Portrait de la santé sexuelle des hommes de la région métropolitaine de Montréal ayant des relations sexuelles avec des hommes Cycle 2017-2018 Faits saillants. Montréal (QC): Direction régionale de santé publique, CIUSSS du Centre-Sud-de-I'Île-de-Montréal; 2019 Jan. https://www.engage-men.ca/wp-content/uploads/2019/03/ engage_faitssaillants_Mars-2019-B.pdf 